\title{
Evaluation of different techniques in management of secondary rhinoplasty cases
}

\author{
Hayam Sabry ${ }^{*}$, Iman L Salem ${ }^{2}$, Naser A Ghozlan ${ }^{2}$ and Ahmed A Zedan ${ }^{2}$ \\ ${ }^{1}$ Department of Plastic Surgery, Faculty of Medicine, Kafrelsheikh University, Egypt \\ ${ }^{2}$ Department of Surgery, Faculty of Medicine, Alexandria University, Egypt
}

\begin{abstract}
Objective: To assess functional and aesthetic features in patients with previous nasal surgery or trauma and evaluate the outcomes of varied techniques.

Methods: The study included 20 patients of both sex presented for secondary rhinoplasty after previous surgery or trauma. They admitted at Plastic and Reconstructive Surgery Unit; Alexandria Main University Hospital who underwent rhinoplasty between 2017 and 2019 . All patients were treated through an open approach by different techniques according to their main problems either aesthetic, functional or both. Our techniques were mainly classified into approaches to the external nose and the nasal septum. NOSE and ROE scores" were used to measure patients' satisfaction. During the follow up periods, evaluation of the surgical result depended on clinical examination, comparison of pre- and post-operative photographs and degree of patients' satisfaction with their aesthetic and functional outcome.

Results: Functionally, 78.5\% suffered nasal obstruction that was mainly caused by septal deviations and nasal valve problems. Aesthetically, the most common deformities of the upper two thirds of the nose included dorsal irregularities (50\%), pollybeak (10\%), dorsal saddle (5\%), and open roof deformity (5\%), whereas the deformities of lower third included tip deformity (75\%). Nasal grafting was necessary in all cases; usually more than 1 type of graft was used in each case. Postoperatively, $87.5 \%$ of the patients, with preoperative nasal obstruction, reported improved breathing; $95 \%$ were satisfied with their aesthetic result; and only 2 cases (10\%) requested further revision to correct minor deformities.
\end{abstract}

Conclusion: Secondary rhinoplasty is a complex and technically challenging topic, yet, in a fare percentage of cases, aesthetic and functional improvement are still possible with a good patient satisfaction.

\section{Introduction}

Secondary rhinoplasty is basically a constant challenge to the nasal surgeon aimed at rebuilding the dorsal and tip support. Secondary rhinoplasty is by definition a reoperation of a nose was previously operated upon by a prior surgeon. It is distinguished from a "revision", which is a reoperation by the same surgeon on his/her patient. Usually, the secondary operation is more extensive than the revision. Secondary rhinoplasty also includes the post traumatic rhinoplasty [1].

Secondary problems can be classified as minor or major defects. Minor defects are often accepted by patients like minor dorsal irregularities or slight asymmetry of the nasal tip. Major defects may result from excessive and asymmetrical resections. The revision rhinoplasty is technically more demanding and its difficulties encouraged depend on the number of previous surgeries, the extent cartilage resections, excessive scarring, decreased vascularity and condition of soft tissue envelope.

As a result of the excessive fibrosis and decreased vascularity, the skin soft-tissue envelope of the nose becomes less contractile and red rapes poorly over the modified bony cartilaginous framework, thus showing less details of any modifications performed [2].

For successful correction of post-traumatic deviated nose, all the anatomic components involved in the deformity should be adequately recognized and surgically realigned [3].

Apart from the technical problems encountered, these patients usually suffer from psychological implications and need more reassurance than they did prior to their first surgery. In addition, the operation is often more complicated, and it is important to acknowledge the patient's complaints [4].

The aim of this study is to assess both preoperative functional and aesthetic features in patients with previous nasal surgery or trauma and evaluate the outcomes of varied techniques correlating preoperative deformities to a suitable corrective method and their effect on patients' satisfaction.

\section{Material and methods}

This is a prospective study included 20 patients of both males and females, presented for secondary rhinoplasty after having previous surgery or trauma. They admitted at Plastic and Reconstructive Surgery Unit; Alexandria Main University Hospital who underwent rhinoplasty between 2017 and 2019.

The patients' problems were classified into aesthetic and functional; the aesthetic problems were further subdivided, anatomically, into

${ }^{\star}$ Correspondence to: Hayam Sabry, Department of Plastic Surgery, Faculty of Medicine, Kafrelsheikh University, Egypt, E-mail: dr.hayamsabry84@gmail.com

Key words: secondary rhinoplasty, post-traumatic, revised nose, septoplasty, nasal grafting, polly-beak deformity, saddle nose

Received: January 27, 2020; Accepted: February 10, 2020; Published: February 14,2020 
deformities affecting the upper two thirds or the lower one third of the nose.

The external rhinoplasty approach was used in all the cases, and, whenever indicated, simultaneous septal surgery was performed. The intraoperative findings were correlated to the presenting deformities in each case and a critical analysis of the corrective methods used was performed [5].

All cases were followed up at two weeks, one month, three months and six months post-operative to evaluate the outcome of the performed procedure. Evaluation included periodic clinical examination, analysis of preoperative and postoperative photographs, and the degree of patients' satisfaction with both their aesthetic as well as functional outcome.

\section{Technical considerations}

Approach to the external nose: A care should be taken to preserve the integrity of the external skin as well as of the internal mucosal lining. The external rhinoplasty approach was used in all cases to provide a wide exposure and allow a more accurate assessment and correction of the underlying problems. Unfortunately, because of the distorted anatomy and the extensive adhesions the dissection and assessment wasn't easy; thus, the incision should be performed very carefully to avoid inadvertent injury of the alar cartilages. The columellar incision was done at the preexisting scar to avoid interrupting the blood supply of the columellar flap.

The dorsal nasal skin was usually found to be more fibrotic and with compromised vascularity. The process of skin flap elevation was difficult especially at areas of bony irregularities. In these cases, the dissection was better deep in subperiosteal plane to avoid any trauma to the skin. It was important also to avoid injury the lining mucosa due to the adhesions between it and the external skin because of missing cartilage secondary to previous excisions.

Approach to the nasal septum: Septal surgery was encouraged either to correct residual septal deviations or to harvest septal cartilage for nasal grafting. In cases with dorsal septal deviations, the septum was accessed dorsally through the external rhinoplasty approach. If the deviation affected the caudal septum, the incision was placed directly on the caudal end of the septal cartilage. The cartilage was then dislocated from the maxillary crest, excised and fixed in the midline by suturing it to the underlying periosteum.

Before correction of bony deviations and the harvesting of septal cartilage, every part of the septum should be palpated to determine the areas in which the cartilage and/or bone were previously excised and to decrease the risk of septal perforation. If the central cartilage was missing, a more ventral approach could be adopted by extending the incision downward over the maxillary crest to expose the thick ventral part of the septal cartilage attached to the maxillary crest. This part of septal cartilage was usually found intact even in cases with near total absence of septal cartilage. Harvesting such a long and thick strut of cartilage is very useful in columellar grafting.

According to each problem, either over-resected or under-resected nose with/without tip deformity, different corrective techniques were used including nasal grafting, suturing and/or osteotomies to finally obtain better results functionally and aesthetically. Also, NOSE and ROE scores were used to measure degree of patients' satisfaction.

\section{Results}

Out of the 20 patients included in this study, 11 were women and 9 men; their mean age was 26.6 years. 10 patients were due to previous rhinoplasty operation and most of them were females (80\%) while other 10 patients were due to postnasal trauma and most of them were males (70\%). As regard revision cases, 8 patients had one previous operation and other two patients had more than one surgery while all post traumatic cases had no previous surgeries.

Functionally, variable degrees of nasal obstruction were found in 9 cases; this was mainly due to deviated nasal septum (45\%), nasal valve problems (55\%) and inferior turbinate hypertrophy (30\%).

The most common aesthetic deformities affecting the upper two thirds of the nose included Polly beak deformity in 2 cases (10\%), dorsal irregularities in 10 cases $(50 \%)$, dorsal saddling in 1 case $(5 \%)$, and open roof deformity in 1 case (5\%). On the other hand, the commonest deformities encountered in the lower third of the nose included tip problems in 15 cases (75\%), supratip problems in 2 cases (10\%), infratip lobule problems in 1 case (5\%) and pedestal problems in 6 cases (30\%).

Septal surgery was performed in conjunction with secondary rhinoplasty in 15 cases (75\%). In these cases, the correction of septal deviation was in 9 cases $(60 \%)$, harvesting of cartilage for nasal grafting in 14 cases $(93 \%)$.

Nasal grafting was necessary in all cases to augment the overresected bony cartilaginous framework and to provide the structural support needed for the nasal dorsum and the nasal tip. Usually more than 1 type of graft was used in each patient. The graft material of choice was autogenous cartilage. The used cartilage was harvested from the nasal septum, the auricle and/or costal cartilage. The most common grafts used were the columellar grafts in 13 patients (65\%). Spreader grafts were used in 11 patients (55\%), dorsal augmentation grafts were done in 4 patients (20\%), tip grafts were in 7 patients $(35 \%)$, and the lateral crural strut grafts with/without batten graft were in 2 patients (10\%).

On evaluating the degree of patient satisfaction, functionally, $87.5 \%$ of the cases with preoperative nasal obstruction (17 cases) reported an improvement in breathing. On the other hand, 95\% (19 cases) were satisfied with their aesthetic improvement.

\section{Discussion}

In this study, there were 9 patients suffered of nasal obstruction, which was most commonly caused by residual septal deviations that mostly affected the dorsal cartilaginous septum in the nasal valve area. In these cases, the septum was approached through the open rhinoplasty approach and was straighten then maintained in its corrected position by splinting spreader and/or buttress graft [7].

The second most common cause of obstruction was nasal valve collapse either internal or external. The nasal valve collapse was found in 11 cases (55\%) and was corrected either by placing a spreader graft/ flap ${ }^{(116)}$ between the septum and the upper lateral cartilage on affected side in cases of internal valve obstruction (10 cases) or by using lateral crural strut grafts/turnover flap of lower lateral cartilages in cases of external valve collapse (2 cases) [8].

The external deformities encountered were usually complex because of the distorted anatomy and excessive scarring resulting from prior surgeries. The wide exposure provided by the open rhinoplasty approach proved to be very helpful in these cases. 
In this study, the most common aesthetic deformities affecting the upper two thirds of the nose included dorsal hump and irregularities in 10 cases $(50 \%)$, pollybeak in 2 cases $(10 \%)$, dorsal saddle and the open roof deformity in one case $(5 \%)$. While the most common aesthetic problem affecting the lower third of the nose was tip deformity (75\%) either ill-defined or mal-projected.

All cases of depressed nasal tip were found to be secondary to inadequate nasal tip support as a result of previous excisions of tip cartilages and/or caudal septal cartilage. In these cases, the tip support was done by splinting to a strong collumellar strut. On the other hand, tip definition was managed by using an onlay-type tip graft [9] (Figures $1-3)$.
In the present study, the ROE and NOSE questionnaires were used to measure the degree of patients' satisfaction. Therefore, it was essential to establish the value of rhinoplasty through objective measures for both form and function in the same patient population [10].

\section{Conclusion}

The results of the current study were encouraging; functionally, $87.5 \%$ of the patients, with preoperative nasal obstruction, reported an improvement in their breathing according to NOSE score, whereas aesthetically, $95 \%$ were satisfied with their cosmetic result on basis of ROE score.

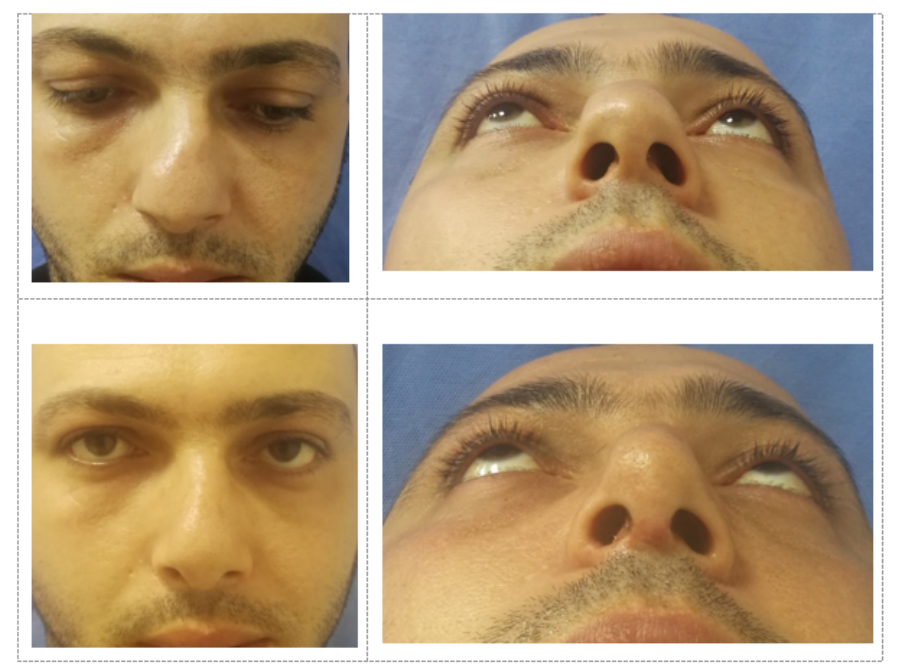

Figure 1. Preoperative views, of a patient who had previous nasal trauma, showing a S-shaped dorsal deviation and depressed left upper lateral cartilage with severe nasal obstruction (above). The basal view shows a broad tip with ill-defined projection. 5 months postoperative views after septorhinoplasty to correct the septal deviation and maintaining its correct position via buttress, bilateral osteotomies, correction of internal valve obstruction by bilateral spreader flaps, tongue-in-groove technique [6] for caudal septum correction and fixation and good tip definition and projection through transdomal and interdomal sutures
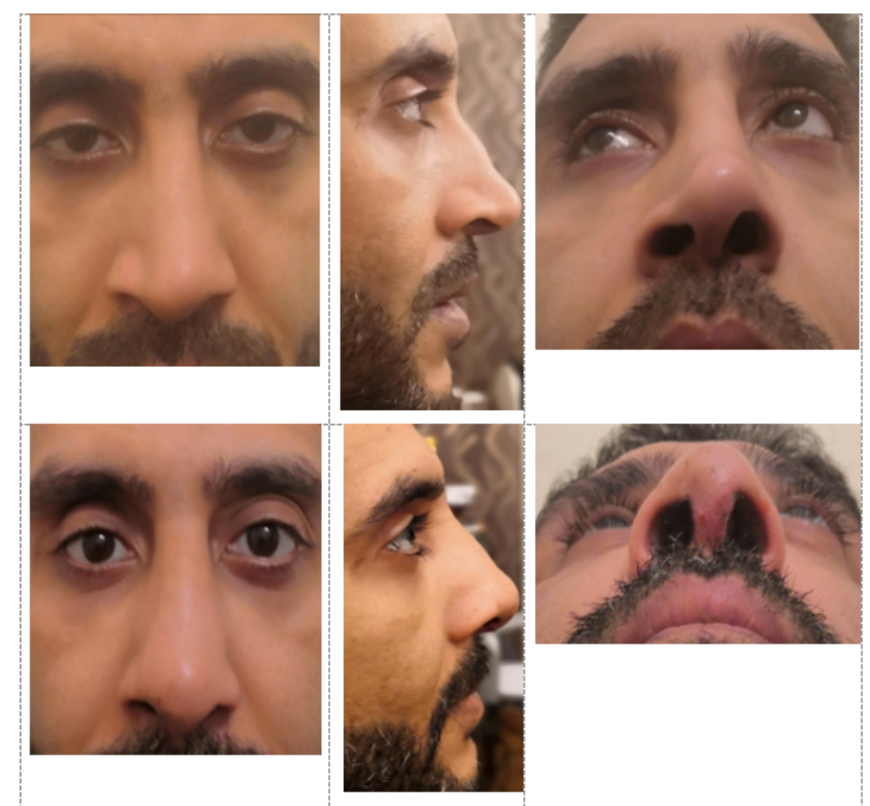

Figure 2. Preoperative views of a patient who had 4 previous rhinoplasties, showing bony dorsal irregularities with moderate nasal skin thickness, open roof deformity with middle third collapse on the right side, right alar collapse leading to domes asymmetry and tip contour deformity, and presented with sever nasal obstruction. The profile view shows a dorsal nasal hump and right alar collapse (above). 3 months postoperative views of the same patient after having an open rhinoplasty with closure of the open roof, rasping of bony irregularities, lowering the cartilaginous dorsum, right spreader grafts, and lateral crural strut grafts on the right side to correct the external nasal valve and nasal obstruction (below) 

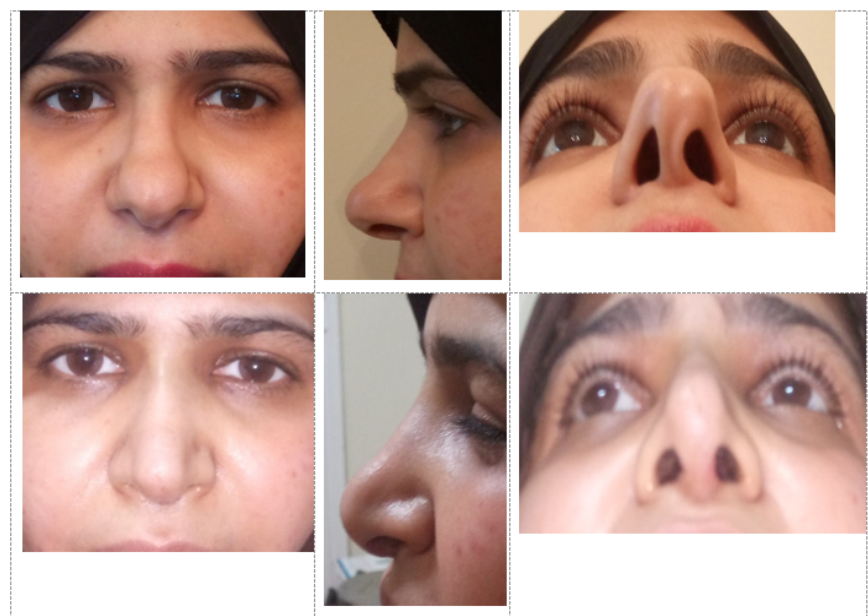

Figure 3. Preoperative views of a patient with tip and nostril deformity. The profile view shows broad splitted nasal domes with poor tip definition (Above). The basal view shows left buckling of medial crura and collapse with nostril asymmerty. Six months postoperative following correction of the left lower lateral cartilage by turnover flap, columellar strut and combining of on-lay tip graft with suturing techniques for more tip projection and definition. No osteotomies were performed

Analysis of NOSE scale showed a significant improvement in the postoperative state compared with the preoperative state with use of a paired $t$ test $(\mathrm{P}<0.01)$. The mean NOSE score preoperatively was $27.50 \pm$ 29.18; the mean postoperative score was $8.75 \pm 10.24$. Whereas analysis of ROE scores showed a significant improvement in the postoperative state compared with the preoperative state $(\mathrm{P}<0.01)$. The mean $\mathrm{ROE}$ score preoperatively was $44.13 \pm 15.85$; the mean postoperative score was $79.56 \pm 14.05$.

\section{References}

1. Byrd HS, Constantian MB, Guyuron B, Pastorek N (2007) Revision rhinoplasty. Aesthet Surg J 27: 175.

2. Aiach GC, Kelly MH (2010) Secondary rhinoplasty. In: Nahai F, ed. The Art of Aesthetic Surgery: Principles and Techniques. $\left(2^{\text {nd }}\right.$ Edn) St. Louis: Quality Medical 1618-1620.

3. Ismail M, Ismail K, Ismail T (2017) The deviated nose: Management and methods for surgical outcomes. Plast Reconstr Surg Glob Open 5: 13.
4. Adams WP Jr, Rohrich RJ, Hollier LH, Minoli J, Thornton LK, et al. (1999) Anatomic basis and clinical implications for nasal tip support in open versus closed rhinoplasty. Plast Reconstr Surg 103: 255-261; discussion 262.

5. Altintas A, Yegin Y, Celik M (2017) Assessment of approaches of otorhinolaryngologists in facial plastic and nasal surgery: A survey study. Turkish Archives of Otorhinolaryngology 55: 129-135.

6. Kridel RWH, Scott BA, Foda HM (1999) The tongue in- groove technique in septorhinoplasty: A ten-year experience. Arch Facial Plast Surg 1: 246-256.

7. Foda HMT (2005) The role of septal surgery in the management of the deviated nose. Plast Reconstr Surg 115: 406-415.

8. Gunter JP, Friedman RM (1997) Lateral crural strut graft: technique and clinical applications in rhinoplasty. Plast Reconstr Surg 99: 943-952.

9. Peck GC (1983) The on lay graft for nasal tip projection. Plast Reconstr Surg 71:27-37

10. McKiernan DC, Banfield G, Kumar R, Hinton AE (2001) Patient benefit from functional and cosmetic rhinoplasty. Clin Otolaryngol Allied Sci 26: 50-52.

Copyright: $(\mathbb{O} 2020$ Sabry H. This is an open-access article distributed under the terms of the Creative Commons Attribution License, which permits unrestricted use, distribution, and reproduction in any medium, provided the original author and source are credited. 